\title{
Introduction to the Minitrack Human-Computer Interaction: Informing Design Utilizing Behavioral, Neurophysiological, and Design Science Methods
}

\author{
Christoph Schneider \\ Department of Information Systems \\ College of Business \\ City University of Hong Kong \\ christoph.schneider@cityu.edu.hk
}

\author{
Joseph S. Valacich \\ Management Information Systems \\ Eller College of Management \\ University of Arizona \\ jsvalacich@cmi.arizona.edu
}

\author{
Angelika Dimoka \\ Fox School of Business \\ Temple University \\ dimoka@temple.edu
}

\section{Introduction}

As we move further into the $21^{\text {st }}$ century, we have witnessed a variety of new and exciting developments that have shaped and will continue to shape the way we interact with information systems. For example, with social media being confined not only to personal use, user interactions with social media take place in both personal and professional settings. The proliferation of mobile devices with a variety of form factors requires examining user interactions beyond a stationary context; the multitude of sensors embedded in mobile devices further enable new forms of interaction, but also open up exciting opportunities for collecting research data. Likewise, people are interacting increasingly with Internet of Things devices; this not only opens up new areas of research, but also enables collecting research data of unprecedented volume, velocity, and variety.

Given vast improvements in processing power and breakthroughs in sensor technologies, researchers in the area of human-computer interaction now have a variety of ways to observe and quantify user behavior and user reactions; many devices (such as various wearable devices) enable collecting data beyond the confines of artificial research laboratories, increasingly enabling researchers to study human-computer interaction phenomena as users interact with devices in natural settings.

Analyzing this big data requires techniques that go beyond what has traditionally been used. At the same time, the use of various data sources, especially in settings outside of traditional research laboratories is likely to introduce various sources of "noise," which poses additional challenges for researchers. In sum, we see tremendous changes and challenges, which will have large implications for not only what is being studied, but also for how data are collected, analyzed, and interpreted.

To address these and other exciting topics and developments in the area of Human-Computer
Interaction (HCI), this minitrack aims to bring together researchers from information systems, computer science, and a broad range of reference disciplines such as design sciences, psychology and neuroscience, as well as bring together academia with industry and government.

\section{Brief History of the Minitrack}

As we continue to witness new and exciting avenues for exploration, the HCI minitrack continues to grow. The minitrack has now existed for more than a decade, and over this time, we have had dozens of great papers presented, many being at the forefront of examining novel phenomena or using novel methodologies.

In 2006, Joe Valacich and John Wells established this minitrack to provide an outlet for a variety of HCI research streams from a variety of disciplines. In 2009, Ryan Wright joined the team, bringing in novel perspectives about personalization and digital security in HCI. In 2012, Angelika Dimoka, an expert in the field of NeuroIS, replaced John Wells. In the subsequent year, we included (for the first time) the disciplines neuroscience and design science, with the aim of Informing Design Utilizing Behavioral, Neurophysiological, and Design Science Methods. In 2014, Christoph Schneider replaced Ryan Wright, bringing a crosscultural perspective into the minitrack.

Our aim has been - and continues to be - to get a truly cross-disciplinary understanding of HCI that informs contemporary research and impacts design practices. Each year, the papers selected for the competitive HCI minitrack draw on this rich crossdisciplinary tradition. Given that HCI continues to grow and change, we aim to provide a forum for the exchange of novel thoughts and ideas. We would like to thank all the authors and reviewers who have supported this mini-track over the years. We look 
forward to another decade of high-quality papers and great interactions during the sessions.

\section{This Year's Minitrack}

For the $50^{\text {th }}$ anniversary of HICSS, we have two sessions with a total of eight papers. The papers in the first session examine user interactions with websites and digital avatars. In the first paper, "Interactive Realistic Digital Avatars-Revisiting the Uncanny Valley," Michael Seymour, Kai Riemer, and Judy Kay examine how embodied conversation agents with realistic human faces challenge the conventional Uncanny Valley theory (namely, that affinity is a function of 'appearance') and introduce a broader theoretical foundation to include interactivity as an additional dimension. In the next paper, titled "Interacting like Humans? Understanding the Effect of Anthropomorphism on Consumer's Willingness to Pay in Online Auctions," Lingyao Yuan and Alan Dennis aim to extend our understanding of online bidding to the area of non-rational decision making and investigate the impact of an automatic processanthropomorphism - on individuals' bidding decisions and what factors can be used to induce this automatic process; results show that visual design induces individuals' anthropomorphism and also impacts bidding decisions. In the third paper, titled "Effects of Color Appeal, Perceived Risk and Culture on User's Decision in Presence of Warning Banner Message," Mario Silic, Dianne Cyr, Andrea Back, and Adrian Holzer examine how color appeal and perceived risk affect users' decision to comply with IT security policies, finding that culture seems to be an important dimension in the specific warning message context in which color appeal is a salient antecedent to behavioral intentions. In the fourth paper of this session, titled "Using Context-Based Password Strength Meter to Nudge Users' Password Generating Behavior: A Randomized Experiment," Warut Khern-am-nuai, Weining Yang, and Ninghui Li use a randomized experiment to show that contextual information could nudge end-users to create stronger passwords.

The second session contains a diverse set of papers related to HCI research and practice. In the first paper, titled "MUX: Development of a Holistic Mobile User Experience Instrument," Soussan Djamasbi and Vance Wilson develop MUX, an instrument for measuring user experiences in mobile computing, which can be used to augment the widely used System Usability Scale to capture characteristics specific to mobile environments. In the second paper, "The Agile UX Development Lifecycle: Combining Formative Usability and Agile Methods," Suzanne Kieffer, Aissa Ghouti, and Benoit Macq present a method and guidelines for the synchronization of UX and agile tasks within agile UX sprints, the identification of relevant UX metrics to be considered, and the improvement of product quality through iterative evaluations. In the third paper, "Using Wearable Devices for Non-invasive, Inexpensive Physiological Data Collection," James Gaskin, Jeffrey Jenkins, Thomas Meservy, Jacob Steffen, and Katherine Payne demonstrate that consumer-grade wearable devices can be used for non-invasive and inexpensive physiological data collection. In the final paper, "Overcoming Innovation Resistance beyond Status Quo Bias-A Decision Support System Approach," Carola Stryja, Verena Dorner, and Lara Riefle present an experimental study about the design of a decision support system (DSS) that addresses innovation resistance to complex innovations on an individual's cognitive level by testing the influence of different DSS modifications on the perception and selection of complex innovations.

We believe that the eight papers presented in the two sessions of this minitrack will provide interesting and thought provoking discussions that will be relevant for both research and practitioners.

We would like to sincerely thank the researchers who submitted their best work to this year's minitrack. Also, we would like to express our thanks for the outstanding efforts put forth by the many reviewers who again helped ensure that the papers presented in this year's minitrack are both interesting and relevant to the HCI field. 